\title{
LATTICE MODES IN THE LINEAR CHAIN COMPOUND ZrT'e
}

\author{
A. Zwick, G. Lenda, R. Carles and M.A. Renucei
}

Laboratoire de Physique des Solides, ^ssocié au C.N.R.S., Université Paul Sabatier 118, Route de Narbonne, 31062 Toulouse Cédex, France

and

A. Kjekshus

Kjemisk Institutt, Universitetet $i$ Oslo

Blindern, Oslo 3, Norway

(Received 13 April 1982 by M. Balkanski)

\begin{abstract}
We report Raman scattering experiments on the linear chain compound $\mathrm{ZrTe}_{5}$. Room and low temperature measurements rule out the existence of a structural phase transition, suggested by the resisitive anomaly near $150 \mathrm{~K}$. The eight $\overrightarrow{\mathrm{k}}=\overrightarrow{0}$ vibrationnal modes allowed by the scattering geometry are observed and their symmetries determined from polarized spectra. Some of the modes are identified on the basis of symmetry properties and (or) in comparison with the Reman spectra of the related compound $\mathrm{ZrTe}_{3}$. Moreover, this comparison indicates surprisingly similar strengths for atomic interactions in $\mathrm{ZrTe}_{3}$ and $\mathrm{ZrTe}_{5}$.
\end{abstract}

Introcuction

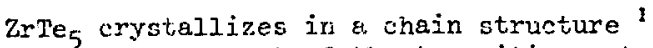
closely reiated to that of the transition-metal trichaicogenides. In some of these low dimensional compounds, sich as $\mathrm{NoSe}_{3}$, a particular form of the Fermi surface produces electronic instebilities winich drive structural phase transitions ${ }^{2,3}$. This explains the recent attention devoted to the pentatellurides $\mathrm{ZrTe}_{5}$ and $\mathrm{HPTe}_{5}{ }^{4}$ and the subsequent investigation of their structura? and transport properties by means of various techniques. In spite of a resistive anomaly " observed near $150 \mathrm{~K}$, the other measurements ${ }^{4}$ ruied out the possibility of an electronicaily-ariven phase transition sinilar to that reported in $\mathrm{NbSe}_{3}$.

In the present paper, we report the results of room and low temperature Raman scattering ex-periments on crystalline $\mathrm{ZrTe}_{5}$. The crystal structure is described in sec. 2. The experinental details are given in Sec. 3. Following the factor group analysis of the crystal and chain $\vec{k}=\vec{\sigma}$ vibrationnal modes, the experimentally observed. spectra are presented and discussed in See. 4.

\section{Crystal Structure}

$\mathrm{ZrTe}_{5}$ was shown to be isostructural with HfTe $_{5}$, the crystal structure of which has been determined by the X-ray work of' Furuseth et al.? ZrTe crystallizes in the Cmem ( 17 ) space group. The conventional non-orimitive unit cell conteins four formula units and has the dimensions $a=$ $3.9876 \AA: b=14.502 \AA$ and $c=13.727 \AA$.

$\mathrm{ZrTe}_{5}$ possesses an interesting structure with both layer and chain features that are res-ponsible for the easy $(010)$ clearage and the $f i-$ brous looking of the crystals. Fig. $T(\mathrm{~B})$ shows projections of the structure into different crystallographic planes, and displayrs the 12-atom primitive unit cell, with two chains passing througn. One can build up the infinite atomic chains paraliel to the a axis by stacking together distorted bicapped trigonal prisms of tellurium with the zirconium atoms located in the centre. The chains are linked by $\mathrm{Te}-\mathrm{Te}$ bonds so as to form layers approximately paralleI to the (010) piane. The layers are held together by weak van der Waals-type forces.

This structure is closely related to that of $\mathrm{ZrTe}_{3}{ }^{6}$ and other $\mathrm{IV}_{\mathrm{b}}$ transition-metal trichalcogenides. The main difference, which accounts for the difference in composition, lies in the linkage between the basic coordination units [see fig. $1(b)$ ].

\section{Experiment}

The samples stuaied in this work were single crystals of $\mathrm{ZrTe}_{5}$, with typical dimensions $6 \times 0,2 \times 0,4 \mathrm{~mm}^{3}$. The Raman measurements were taken on freshly cleaved (010) surfaces in order to avoid scattering from $\mathrm{Te}$, left behind by surface oxidation. The prepared samples were immediately immersed in the exchange gaz of an Oxford CF 204 crycstat for room and low temperature experinents.

The Raman spectra of $\mathrm{ZrTe}_{5}$, excited with the $5145 \AA$ and $5309 \AA$ lines of Spectra Physics argon and krypton ion lasers, were measured in the backscattering geometry. The laser beam was focused onto the surface sample et nearly normal incidence. The scattered light was collected along the crystal $\mathrm{o}$ axis direction and analyzed in a T800 Coderg triple monochromator, in conjunction with standard photoncounting electronics. 

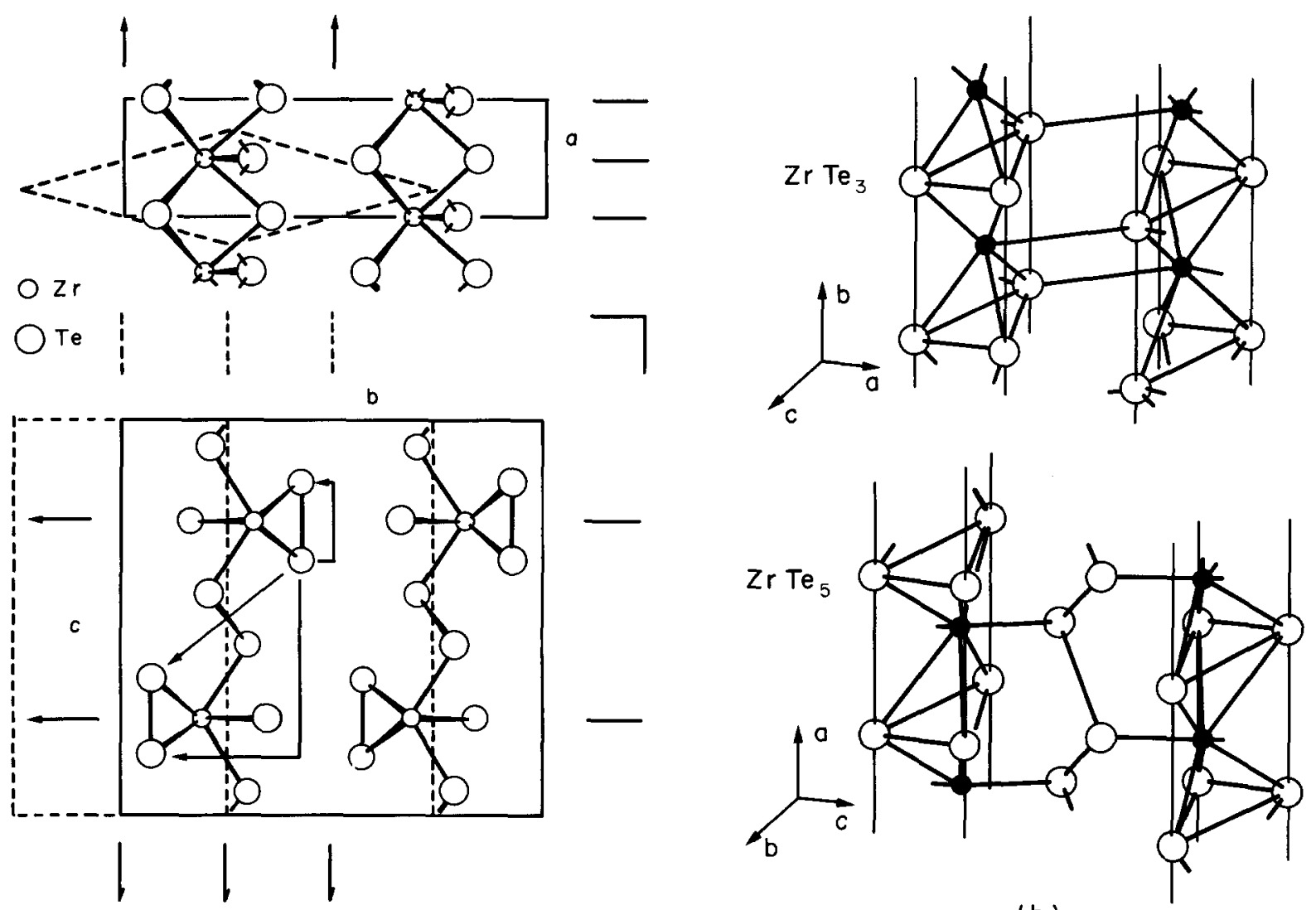

(a)

(b)

Fig. 1. Structure of $\mathrm{ZrTe}_{\text {type crystals }}$

(a) Projections in the ( 801$)$ and (100) planes. The primitive unit cell is shown on the top diagram. The crystal-symmetry elements are indicated on both diagrams.

(b) Interchain linkage in $\mathrm{ZrTe}_{5}$, compared to that in $\mathrm{ZrTe}_{3}$. The principal axes $X, Y, Z$ are chosen to coincide with the crystallographic axes $a, b, c$.

\section{Results and Discussion}

\subsection{Factor group analysis}

The crystal factor-group contains the eight representative symmetry elements :

1 : the identy,

$2_{a}, 2_{b}$ : twofold axes parallei respectively

to the $a$ and $b$ axes,

$2_{c}$ : a twofold screw axis parallel to $c$,

$\overline{1}$ : a centre of symmetry,

$\overline{2}_{e}, \overline{2}_{c}$ : mirror planes perpendicular respectively to $a$ and $c$,

$\bar{T}_{b}$ : a glide plene perpendicular to $b$.

Four of these symmetry elements (the identi$t y$, the $2_{\mathrm{b}}$ twofold axis and the $\overline{\overline{2}}, \overline{2}$ mirror planes) are in common with the factor group of a single chain.

The symetries of the Iong-wavelength phonons are determined by the representation $\Gamma_{\text {crystal }}$ of the crystal factor group generated by the displacernents of the atoms in tie primitive unit cell.
$\Gamma_{\text {crystal }}$ is 36 dimensional for the 12-atom primitive unit cell of ZrTes. The reduction of $\Gamma_{\text {crystal }}$ into irreducible representations of the isomorphic point group mm $\left(D_{2 h}\right)$ is as follows :

$$
\begin{aligned}
I_{\text {erystal }}= & 6 A_{g}+6 B_{3 g}+2 B_{2 g}+4 B_{1 g}+6 B_{2 u} \\
& +2 A_{u}+4 B_{3 u}+6 B_{1 u}
\end{aligned}
$$

Because of the inversion operation contained in the crystal factor-group, Raman and infra-red activities are mutually exclusive. The eighteen even-parity modes are optical Raman active phonons, while the eighteen odd-parity modes consist of three acoustical and fifteen optical infra-red active phonons. The $\vec{k}=\overrightarrow{0}$ modes can further be classified according to atomic displacements, parallel to the chains (or out-of- $\overline{\mathrm{C}}_{\mathrm{a}}$ mirror plane) for $\mathrm{B}_{1 g}, \mathrm{~B}_{2 \mathrm{~g}}, \mathrm{~B}_{3 \mathrm{u}}, \mathrm{A}_{u}$ symmetries and perpendicular to the chains (or in $-\bar{c}_{a}$ mirror plane) for $A_{g}: B_{3 z}, B_{2 u}, B_{1 \mu}$.

Since the chains constitute well-defined 
lnits in the crystal, it is convenient to first look at the long-wavelength modes of a single chain and then establish a compatibility relationship connecting the chain to the crystal. The chain factor-group representation $\Gamma_{\text {chain }}$ Generated by the displacenents of atoms in the 6-atom primitive unit cell is 18 dimensional, and reducible into irreducible representations of the isomorphic point group $2 \mathrm{~mm}\left(\mathrm{C}_{2 \mathrm{~V}}\right)$ :

$$
\Gamma_{\text {chain }}: 6 \mathrm{~A}_{1}+6 \mathrm{~B}_{1}+2 \mathrm{~A}_{2}+4 \mathrm{~B}_{2}
$$

Due to the lack of a centre of symmetry, the long-wavelength optical modes of the chain are not aivided into even -and odd- symmetry types. The chains modes can be classified accorjing to atomic displacements in -or out- of $\overline{2}_{a}$ mirror plane, corresponding respectively to indices 1 and 2 . A and $B$ stands for modes respectively symetric and antisymetric with respect to the twofold rotation. The correlation method was used to relate the irreducible representations of the zirconium and tellurium atoms site groups to those of the chain factor group. From the correlation chart presented in table $I(a)$, we can determine which atoms move in each normal mode and wether this motion is along or perpendicular to the chain axis.

Table I(b) displays the correlation diagram relating the long-wavelength chain and crystal thonons in $\mathrm{ZrTe}_{5}$. Since there are two chains per primitive unit cell correlated via an inversion centre, each chain mode splits into a $\mathrm{g}^{-\mathrm{u}}$ pair in the crystal. Furthermore, because the crystal reteins all the symmetry elements of the chain, there is no mixing of the chain modes symmetries in the crystal.

In particular, the $A$ and $B$ symmetries do not mix, in contrast to what happens in the monocliric structure of the $I V_{b}$ metal-transition trichalcogenides. Lastly, as a result of the interchain coupling, the $A_{1}+2 B_{1}+B_{2}$ zero frequency modes of the chain divide up into four odd crystal modes (three acoustical $\mathrm{B}_{2 \mathrm{u}}+\mathrm{B}_{1 \mathrm{u}}+\mathrm{B}_{3 \mathrm{u}}$ and one infra-red $B_{1 u}$ ) and four low-lying even modes $A_{B}+2 B_{35}+B_{1 g}$. These Raman active modes are expected to be lägely rigid chain motions. They can be classified in three translational modes $\mathrm{B}_{1 g}, \mathrm{~A}_{\mathrm{g}}, \mathrm{B}_{3 \mathrm{~g}}$ respectively along the $\mathrm{a}-, \mathrm{b}-$ and $c$ axes, and a librationnal mode $B_{3 g}$ about the a

\subsection{Experimental results and discussion}

In the XYZ set of principal axes, chosen sc as to coincide with the abc crystallographic axes of the crystal, the poiarizability tensors 7 of the $\vec{k}=\vec{d}$ Ranan-active modes of $Z^{\prime} T^{\prime} e_{5}$ have the form :

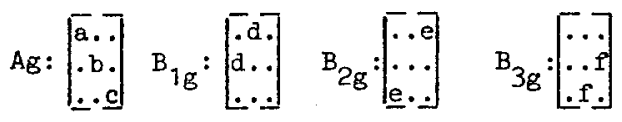

The backscattering geometry described in 3 allows the measurements of Raman tensor components $X X, Z Z, X Z$. Thus $\operatorname{six} A_{g}$ and two $B_{2 g}$ modes should be experimentally obsérved among the eighteen modes theoretically predicted.

Fig. 2 shows unanalyzed Raman spectra for two temperatures, above and below $150 \mathrm{~K}$. As can be seen, eight peaks are clearly observed at room temperature. No new peak occurs at low temperature that would reveal any change of the crystallographic unit cell, such as those induced by charge density waves in many layered transition dichalcogenides 8,9 .

The different components of Raman tensor allowed by the scattering geometry are shown in Fig. 3, which depicts modes polerized respectively parallel $\left(\mathrm{B}_{2 \mathrm{~g}}\right)$ and perpendicular $\left(\mathrm{A}_{\mathrm{g}}\right)$ to the chains. The symmetries and wavenumbers of the observed $\vec{k}=\overrightarrow{0}$ Raman active modes are given in Table II.

We shall approach the mode assignment from the analysis of the single chain vibrations, substantiated by a comparison with the Raman spectra of $\mathrm{ZrTe}_{3}$. We present in Table III the list of $\vec{k}=\overrightarrow{0}$ phonons in $\mathrm{ZrTe}_{3}$ and their assignment to chain modes accoraing to the Ramar works of Zwick et al.10, and Wieting et al.11.

As it appears from the comparison of Table III, the longwavelength phonons of $\mathrm{ZrTe}_{5}$ lye very near in frequency to those of $2 r T e_{3}$. Beyond the close relation between the two structures, this provides evidence of similar strengths for atomic interactions in the two compounds.

Considering first in $\mathrm{ZrT}_{5}$ the two $\mathrm{B}_{2}$ modes polarized along the a axis, we see from Toble I(b) that they originate from $A_{2}$ chain modes. Table $I(a)$ shows that these $A_{2}$ modes aro mixtures of shearing motions of the TeIT and TeIIT pairs respectively. In the crude model of bond stretching forces between nearest neighbours in the chain, the shearing motion of Te atoms does not imply any stretching of the Zr-TeIII bonds, which are perpendicular to the chains. The interchain coupling rises this zero frequency mode until it mixes with the shearing mode of the TeII pair, which depends essentially on intrachain $\mathrm{Zr}-\mathrm{Te}$ II interaction. Assuming the same interaction between $\mathrm{Zr}$ and TeII atoms within the chains in $\mathrm{ZrTe}_{3}$ and $\mathrm{ZrTe}_{5}$, we expect in $\mathrm{ZrTe}_{5}$ a $\mathrm{B}_{2 \mathrm{~g}}$ crystal doublet, very close in frequency to the $\mathrm{B}_{\mathrm{g}}$ mode of $\mathrm{ZrTe}_{3}$ originating from the shearing of the Te pair in the chain. The $\mathrm{B}_{2 \mathrm{~g}}$ spectrum of $2 \mathrm{rTe}_{5}$ (Fig. 3 ) exhibits clearly a doublet of lines lying respectively at 74 and.
$89.5 \mathrm{~cm}^{-1}$, close in frequency to the single $B_{g}$ mode observed at $68 \mathrm{~cm}^{-1}$ in $\mathrm{ZrTe}_{3}$. The Raman study of $\mathrm{ZrTe}_{5}$ thus substantiates in turn mode assignment in $\mathrm{ZrTe}_{3}$ : we confidently attribute to $\mathrm{A}_{2}$ chain mode the $68 \mathrm{~cm}^{-1}$ line in $\mathrm{ZrTe}_{3}$, in agreement with previous assignment by Wieting and coworkers 11 based on the line intensity.

Looking now at the six $\mathrm{A}_{\mathrm{g}}$ modes of $\mathrm{ZrTe}_{5}$, we refer back to Table $I(b)$ and conclude that they all proceed from $A_{1}$ chain modes. According to theoretical expectations, we assign the lowest lying line at $39 \mathrm{~cm}^{-1}$ to the quasi-rigid translationnal motion of the chains along the b-axis. Its wavenumber is surprinsingly close to that of the lowest $\mathrm{A}_{\text {g }}$ mode in $\mathrm{ZrTe}_{3}\left(37 \mathrm{~cm}^{-1}\right)$, which

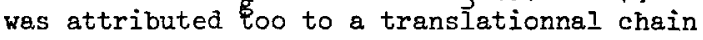
mode of the crystal. This might prove in turn the $A_{1}$ symmetry of the $37 \mathrm{~cm}^{-1}$ line of $2 \mathrm{rTe}_{3}$, as we proposed in earlier work 10 . The highest frequency line at $239 \mathrm{~cm}^{-1}$ is attributed to the stretching mode of the diatomic (TeII) 2 "molecule". This mode lies at slightly higher frequency than the corresponding mode of $\mathrm{ZrTe}_{3}\left(217 \mathrm{~cm}^{-1}\right)$, that would indicate stronger atomic interaction and shorter covalent bond length between the pairing $\mathrm{Te}_{\text {II }}$ atoms in $\mathrm{ZrTe}_{5}$ than in $\mathrm{ZrTe}_{3}$. As for the others $A_{g}$ lines, such a qualitative discussion 
Table I.

(a) Correlation chart between $C_{\partial V}$ and $C_{S}$ site groups of $\left(\mathrm{Zr}, \mathrm{Te}_{I}\right)$ and $\left(\mathrm{Te} \mathrm{e}_{I I}, \mathrm{Te}_{I I I}\right)$ atoms and $\mathrm{C}_{2 \mathrm{~V}}$ chain factor group in $\mathrm{ZrTe}_{5}$.

(b) Compatibility diagram relating the chain and crystal vibrations of $\mathrm{ZrTe}_{5}$.

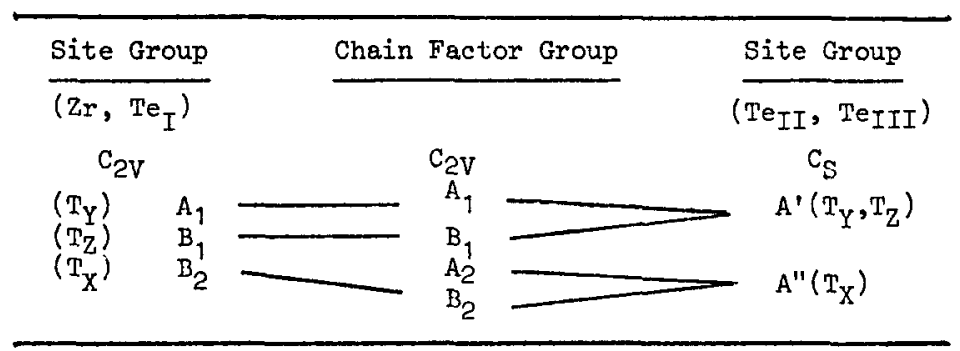

(a)

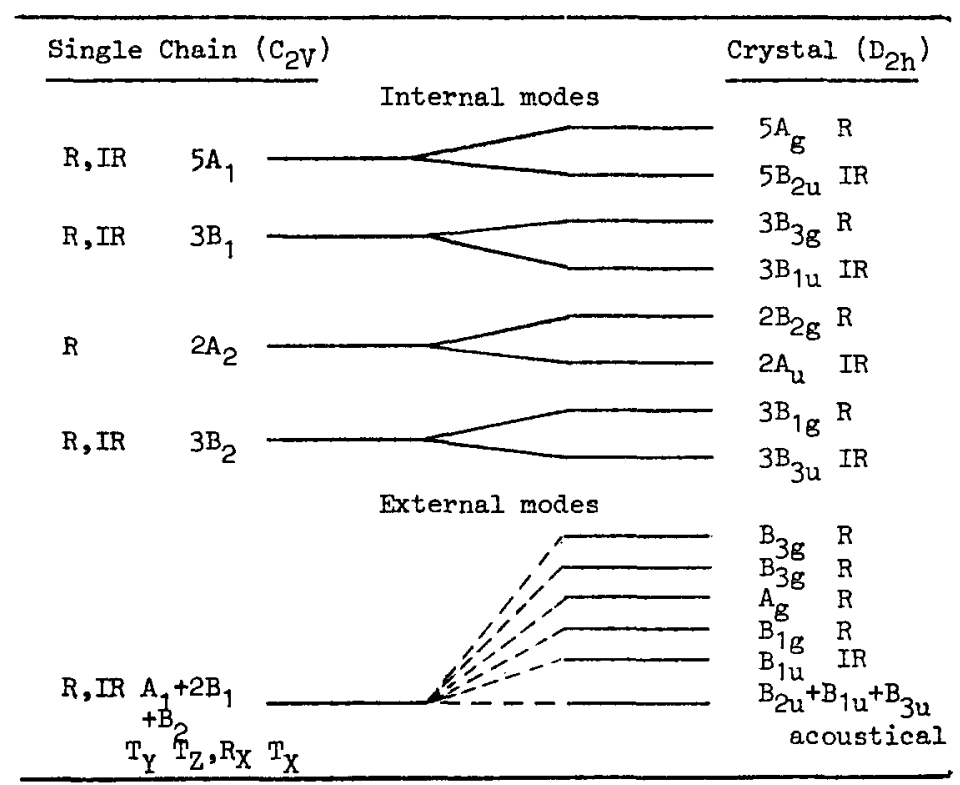

(b)

Table II. Wavenumbers and symetries of $\vec{k}=\overrightarrow{0}$ Raman active phonons of $\mathrm{ZrTe}_{5}$ at $300 \mathrm{~K}$ and $77 \mathrm{~K}$.

\begin{tabular}{|c|c|c|c|c|c|c|c|c|c|}
\hline \multirow{2}{*}{ Symmetry } & Chain & $A_{1}$ & $A_{2}$ & $A_{2}$ & $A_{1}$ & $A_{1}$ & $A_{1}$ & $A_{1}$ & $A_{1}$ \\
\hline & Crystal & $A_{g}$ & $B_{2 g}$ & $\mathrm{~B}_{2 \mathrm{~g}}$ & $A_{B}$ & $\mathrm{~A}_{\mathrm{g}}$ & $A_{g}$ & $\mathrm{~A}_{\mathrm{G}}$ & $\dot{A}_{G}$ \\
\hline \multirow{2}{*}{$v\left(\mathrm{~cm}^{-1}\right)$} & $300 \mathrm{~K}$ & 39 & 72 & 86.5 & 116 & 121 & 147 & 181.5 & \\
\hline & $77 \mathrm{~K}$ & 40 & 74 & 89.5 & 117 & 123 & 152 & 183.5 & 239 \\
\hline
\end{tabular}




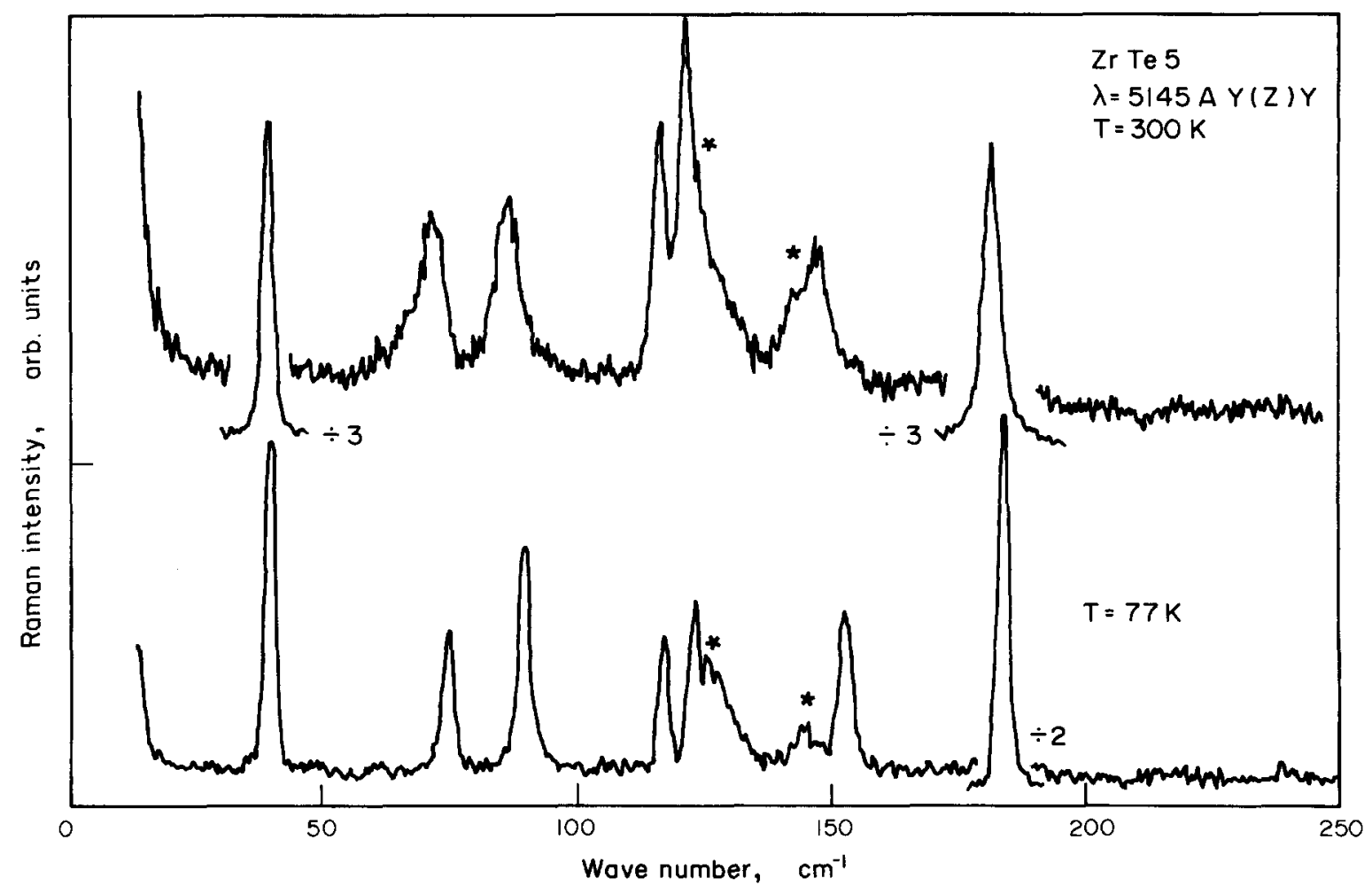

Fig.2. Unpolarized Raman spectra of $\mathrm{ZrTe}_{5}$ excitea with $\lambda=5 i 45 \AA$ at $300 \mathrm{~K}$ and $77 \mathrm{~K}$. The structures marked by an asterisk are due to scattering by $A_{1}$ and $E$ modes of crystalline $\mathrm{Te}$, left behind by surface oxidation.

Table III. Wavenumbers and symmetries of $\vec{k}=\overrightarrow{0}$ Ramen active phonons of $\mathrm{ZrTe}_{3}$ at $300 \mathrm{~K}$.

\begin{tabular}{|c|c|c|c|c|c|}
\hline \multirow{2}{*}{\multicolumn{2}{|c|}{$\frac{\text { Ref. } 11}{\text { Syminetry }}$}} & & \multicolumn{3}{|c|}{ Ref. 10} \\
\hline & & \multirow{2}{*}{$\nu\left(\mathrm{cm}^{-1}\right)$} & \multicolumn{2}{|c|}{ Symmetry } & \multirow{2}{*}{$v\left(\mathrm{~cm}^{-1}\right)$} \\
\hline Crystal & Chain & & Crystal & Chain & \\
\hline$A_{g}$ & $\mathrm{~B}_{1}\left(\mathrm{~T}_{X}\right)$ & 11 & & & \\
\hline$A_{g}$ & $\mathrm{~B}_{1}\left(\mathrm{R}_{\mathrm{Y}}\right)$ & 38 & $A_{B}$ & $A_{1}\left(T_{z}\right)$ & 37.5 \\
\hline$A_{B}$ & $A_{1}\left(T_{Z}\right)$ & 62 & $A_{g}$ & $B_{1}\left(T_{X}\right)$ & 61.5 \\
\hline $\mathrm{B}_{\mathrm{g}}$ & $\mathrm{A}_{2}$ & 64 & $\mathrm{~B}_{\mathrm{g}}$ & & 66.5 \\
\hline$A_{g}$ & $\mathrm{~B}_{1}$ & 86 & $\mathrm{~A}_{\mathrm{g}}$ & $B_{1}\left(R_{Y}\right)$ & 84.5 \\
\hline$A_{g}$ & $A_{1}$ & 108 & $A_{g}$ & $A_{q}$ & 111 \\
\hline$A_{g}^{5}$ & $A_{1}$ & 144 & $A_{g}$ & $A_{1}$ & 143.5 \\
\hline$A_{g}$ & $A_{1}$ & 216 & $A_{g}$ & $A_{1}$ & 215 \\
\hline
\end{tabular}




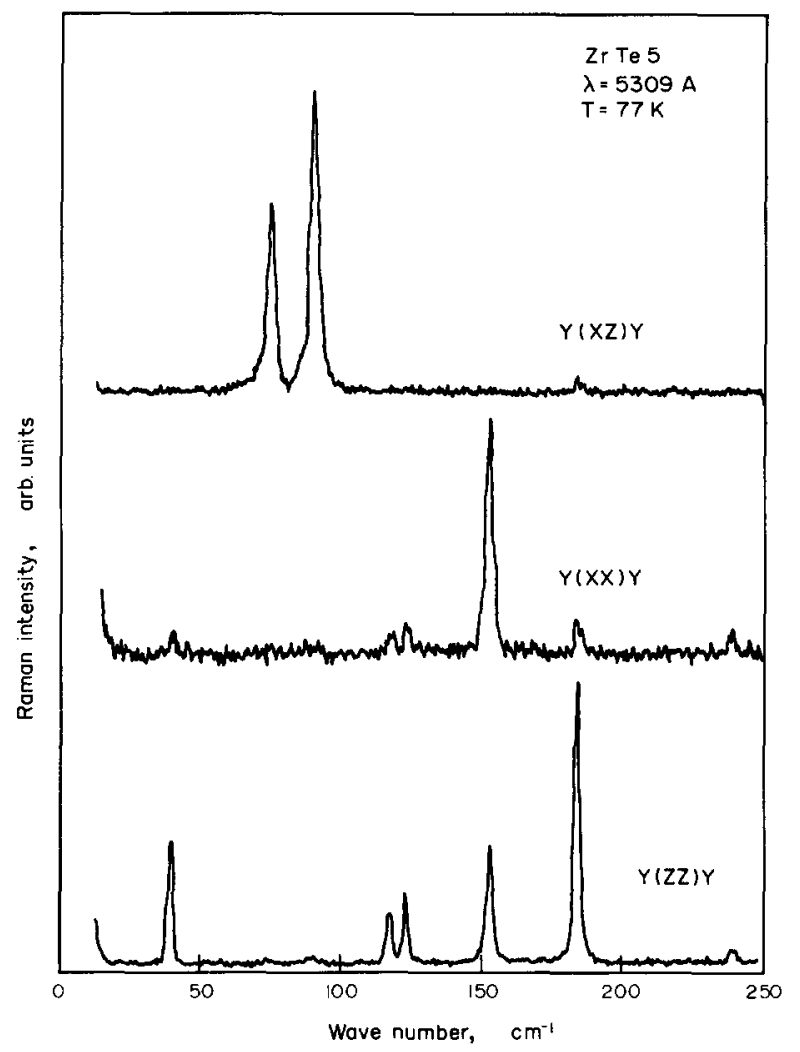

Fig.3. Polarized Raman spectra of $\mathrm{ZrTe}_{5}$ excited with $\lambda=5309 \AA$ at $77 \mathrm{~K}$.

is not sufficient, and an interatomic-force model is needed to describe the lattice dynamics of $\mathrm{ZrTe}_{5}$ and calculate the eigenvectors of the corresponding modes.

\section{Conclusion}

The Raman study of crystalline $\mathrm{ZrTe}_{5}$ at room and liquid nitrogen temperatures rules out the possibility of an electronically driven phase transition near $150 \mathrm{~K}$ suggested by a resistive anomaly. AII the $\vec{k}=\overrightarrow{0}$ phonons predicted by group theory analysis and allowed by the scattering geometry have been observed, and their symmetry determined by polarization measurements. An approach of the crystal lattice dynamics from the "molecular" point of view allows the identification of some crystal modes, in absence of complete calculation based on an interatomic-force model. The comparison with the Raman spectra of the closely related compound $2 \mathrm{rTe}_{3}$ demonstrates that, in spite of the dissimilarity in internal architecture of the basic layers, the strengths of atomic interactions are similar in the two compounds.

\section{References}

1 S. Furuseth, L. Brattás and A. Kjekshus, Acta Chem. Scand. 27, 2367 (1973).

2 N.P. Ong and P. Monceau, Phys. Rev. B16, 3443 (1977).

3 R.M. Fleming, D.E. Moncton and D.B. MeWhan, Phys. Rev. B15, 5560 (1976).

4 F.J. DiSalvo, R.M. Fleming and J.V. Waszczak, Phys. Rev. B24, 2935 (1981).

5 T.J. Wieting, D.U. Gubser, S.A. Wolf and F. Levy, Buzz. Am. Phys. Soc. 25, 340 (1980).

- S. Furuseth, L. Brattas and A. Kjekshus, Acta Chem. Scand. A29, 623 (1975).
7 R. Loudon, Adv. Phys. 13, 423 (1964).

J.R. Duffey, R.D. Kirby and R.V. Coleman, Solid State Commun. 20,617 (1976).

- J.E. Smith, J.C. Tsang and M.W. Shafer, Solid State Commun. 19, 283 (1976).

10 A. Zwick, M.A. Renucci and A. Kjekshus, J. Phys. C Solid. State Phys. 13, 5603 (1980).

11 T.J. Wieting, A. Grisel and F. Lévy, Physica 105B, 366 (1981). 